\title{
Growth Optimal Portfolio Selection Under Proportional Transaction Costs with Obligatory Diversification
}

\author{
T. Duncan • B. Pasik Duncan · L. Stettner
}

Published online: 31 July 2010

(C) The Author(s) 2010. This article is published with open access at Springerlink.com

\begin{abstract}
A continuous time long run growth optimal or optimal logarithmic utility portfolio with proportional transaction costs consisting of a fixed proportional cost and a cost proportional to the volume of transaction is considered. The asset prices are modeled as exponent of diffusion with jumps whose parameters depend on a finite state Markov process of economic factors. An obligatory portfolio diversification is introduced, accordingly to which it is required to invest at least a fixed small portion of our wealth in each asset.
\end{abstract}

Keywords Markov process · Bellman equation · Penalty equation · Impulsive control $\cdot$ Portfolio optimization

\section{Introduction}

Assume that on a given complete probability space $\left(\Omega, \mathcal{F},\left(\mathcal{F}_{t}\right), P\right)$, there are three independent processes adapted to the filtration $\left(\mathcal{F}_{t}\right): d$-dimensional standard Brownian motion $\left(B_{t}, t \geq 0\right)$, a $d$-dimensional compensated Poisson random measure $\tilde{N}(d t, d y)$ and a time homogeneous Markov process $\left(z_{t}, t \geq 0\right)$ with values in a finite

L. Stettner's research was supported by MNiSzW grant no. N N201 371836.

T. Duncan · B. Pasik Duncan

Department of Mathematics, University of Kansas, Lawrence, KS 66045, USA

T. Duncan

e-mail: duncan@math.ku.edu

B. Pasik Duncan

e-mail: bozenna@math.ku.edu

L. Stettner $(\bowtie)$

Institute of Mathematics Polish Acad. Sci., Sniadeckich 8, 00-956 Warsaw, Poland

e-mail: stettner@impan.gov.pl 
space $D$ and a transition matrix $P_{t}$ at time $t$. Consider also $d$ assets with the $i$ th asset price denoted $S_{i}(t)$ at time $t$. It is assumed that the evolution of $S_{i}(t)$ is of the form

$$
S_{i}(t)=S_{i}(0) e^{X_{i}(t)}
$$

where $X_{i}(0)=0$ and $X(t)=\left(X_{1}(t), \ldots, X_{d}(t)\right)^{T}$ with the superscript $T$ denoting transpose, is a solution to the following Lévy stochastic differential equation:

$$
d X(t)=a\left(z_{t}\right) d t+\sigma\left(z_{t}\right) d B_{t}+\int_{R^{d}} \gamma\left(z_{t}, y\right) \tilde{N}(d t, d y) .
$$

It is assumed that $a, \sigma$, and $\gamma$ are functions of a parameter $z$ from the finite space $D$ and $\gamma_{i k}\left(z, y_{k}\right)$ depends on the $k$-th coordinate $y_{k}$ of $y \in R^{d}$

$$
\int_{R^{d}}\left|\gamma_{i k}\right|^{\iota}\left(z, y_{k}\right) v_{k}\left(d y_{k}\right)<\infty
$$

for $\iota=1,2, i, k=1,2, \ldots, d$, with $v_{k}$ being the Lévy measure corresponding to $\tilde{N}(d t, d u)$. Let $W_{t}$ be the wealth process at time $t$ and let $\pi_{i}(t)$ be the portion of $W_{t}$ invested in the $i$ th asset. Let $e^{X(t)}=\left(e^{X_{1}(t)}, \ldots, e^{X_{d}(t)}\right)$ and for $\pi, \zeta \in[0, \infty)^{d} \backslash$ $\{(0, \ldots, 0)\}$

$$
\begin{aligned}
\pi \diamond \zeta & =\left(\pi_{1} \zeta_{1}, \pi_{2} \zeta_{2}, \ldots, \pi_{d} \zeta_{d}\right) \\
\pi \cdot \zeta & =\pi_{1} \zeta_{1}+\pi_{2} \zeta_{2}+\cdots+\pi_{d} \zeta_{d}
\end{aligned}
$$

and

$$
g(\zeta)=\left(\frac{\zeta_{1}}{\sum \zeta_{i}}, \frac{\zeta_{2}}{\sum \zeta_{i}}, \ldots, \frac{\zeta_{d}}{\sum \zeta_{i}}\right) .
$$

If the portfolio strategy in the time interval $[0, t]$ is not changed then the wealth process and the portions of the wealth invested in the assets at time $t$ are of the form

$$
W_{t}=W_{0} \pi(0) \cdot e^{X(t)}, \quad \pi(t)=g\left(\pi(0) \diamond e^{X(t)}\right) .
$$

By the form of (2), it is clear that the pair $\left(\pi(t), z_{t}\right)$ and the triple $\left(\pi(t), z_{t}, W_{t}\right)$ are Markov processes with transition operators $\Pi_{t}$ and $\Pi_{t}^{e}$ respectively.

Let $S=\left\{v=\left(v_{1}, \ldots, v_{d}\right), v_{i} \geq 0, \sum v_{i}=1\right\}, S^{0}=\left\{v=\left(v_{1}, \ldots, v_{d}\right), v_{i}>0\right.$, $\left.\sum v_{i}=1\right\}, S_{\delta}=\left\{v \in S, v_{i} \geq \delta, i=1,2, \ldots, d\right\}$ and $S_{\delta}^{0}=\left\{v \in S, v_{i}>\delta, i=\right.$ $1,2, \ldots, d\}$, for $0<\delta<1 / d$.

Assume that there are proportional transaction costs consisting of a fixed proportional managing cost and a cost proportional to the volume of the transactions. Let $\zeta_{i}^{-}, i=1,2, \ldots, d$ denote the amount of wealth process invested in the $i$ th asset before a possible transaction. Clearly, $W^{-}=\sum_{i=1}^{d} \zeta_{i}^{-}$is the wealth before a possible transaction. The change of the portfolio to $\left(\zeta_{1}, \zeta_{2}, \ldots, \zeta_{d}\right)$ requires paying immediately transaction costs of the form

$$
\kappa W^{-}+\sum_{i=1}^{d} c_{i}^{1}\left(\zeta_{i}-\zeta_{i}^{-}\right)^{+}+c_{i}^{2}\left(\zeta_{i}-\zeta_{i}^{-}\right)^{-}
$$


with $\kappa>0$ corresponding to a fixed managing cost (see [10] for a justification). Short selling or short borrowing are not allowed and it is assumed that the portfolio is selffinancing. Therefore, the wealth $W$ after the transaction is equal to

$$
W^{-}-\kappa W^{-}-\sum_{i=1}^{d} c_{i}^{1}\left(\zeta_{i}-\zeta_{i}^{-}\right)^{+}+c_{i}^{2}\left(\zeta_{i}-\zeta_{i}^{-}\right)^{-}
$$

Let $\pi_{i}^{-}=\zeta_{i}^{-} / W^{-}$and $\pi_{i}=\zeta_{i} / W$ be respectively the portion of wealth invested in the $i$ th asset before and after a transaction. From (5), it follows that

$$
1-\kappa-\sum_{i=1}^{d} c_{i}^{1}\left(\pi_{i} \frac{W}{W^{-}}-\pi_{i}^{-}\right)^{+}+c_{i}^{2}\left(\pi_{i} \frac{W}{W^{-}}-\pi_{i}^{-}\right)=\frac{W}{W^{-}}
$$

or

$$
c\left(\pi \frac{W}{W^{-}}-\pi^{-}\right)+\frac{W}{W^{-}}=1,
$$

with

$$
c(v)=\kappa+\sum_{i=1}^{d}\left(c_{i}^{1} v_{i}^{+}+c_{i}^{2} v_{i}^{-}\right) .
$$

In what follows, it is assumed that $0<c_{i}^{1}, c_{i}^{2}<1-\kappa$.

It appears that starting from portfolio $\left(\pi_{1}^{-}, \pi_{2}^{-}, \ldots, \pi_{d}^{-}\right)^{T}$ any portfolio $\left(\pi_{1}\right.$, $\left.\pi_{2}, \ldots, \pi_{d}\right)^{T} \in S$ is available. Naturally it follows that (see Lemma 1 of [15], or [14])

Lemma 1 There is a unique continuous function $e: S \times S \rightarrow(0,1-\kappa]$ such that for $\pi^{-}, \pi \in S$ there is the equality

$$
c\left(\pi e\left(\pi^{-}, \pi\right)-\pi^{-}\right)+e\left(\pi^{-}, \pi\right)=1 .
$$

The function e is bounded away from zero and

$$
e\left(\pi, \pi^{\prime}\right) e\left(\pi^{\prime}, \pi^{\prime \prime}\right)<e\left(\pi, \pi^{\prime \prime}\right)
$$

which means that it is not profitable to make two instantaneous portfolio changes. The wealth process $W^{-}$after the change of portfolio from $\pi^{-}$to $\pi$ is diminished to $W=e\left(\pi^{-}, \pi\right) W^{-}$.

Denote by $W_{t}^{-}, W_{t}, \pi^{-}(t), \pi(t)$, the wealth process before and after transaction or the portfolio before and after transaction at time $t$ respectively. The purpose is to maximize the following long run wealth growth rate or in other words long run logarithmic utility from terminal wealth:

$$
J\left((\pi(s))=\liminf _{t \rightarrow \infty} \frac{1}{t} E_{\pi z}\left[\ln W_{t}\right]\right.
$$


Since $\kappa>0$, the strategy is of impulsive form - i.e. it is a sequence $V=\left(\tau_{n}, \pi^{n}\right)$ consisting of transaction times (stopping times $\tau_{n}$ for $n=1,2, \ldots$ ) and portfolios $\pi^{n}$ which are chosen at time $\tau_{n}$. The following equalities are satisfied

$$
\begin{aligned}
& W_{t}=W_{\tau_{n}} \sum_{i=1}^{d} \pi_{i}\left(\tau_{n}\right) e^{X_{i}(t)-X_{i}\left(\tau_{n}\right)} \\
& \pi(t)=g\left(\pi\left(\tau_{n}\right) \diamond e^{X(t)-X\left(\tau_{n}\right)}\right)
\end{aligned}
$$

for $\tau_{n}<t<\tau_{n+1}$, and

$$
W_{\tau_{n}}=e\left(\pi^{-}\left(\tau_{n}\right), \pi^{n}\right) W_{\tau_{n}}^{-} .
$$

Additionally the portfolio $\pi(t)$ is not allowed to be too close to the boundary of the simplex $S$. We introduce an obligatory diversification of the portfolio. Let $0<\delta<\delta^{\prime}<1 / d$. As soon as the portfolio $(\pi(t))$ enters the set $S \backslash S_{\delta}^{0}$, it is changed by choosing a new portfolio from the set $S_{\delta^{\prime}}$. Both parameters $\delta$ and $\delta^{\prime}$ are assumed to be fixed in this paper. The following remark justifies the use of obligatory portfolio diversification:

Remark 1 Assume that there is a unique invariant measure $\mu$ for Markov process $\left(z_{t}\right)$. Under the assumptions, the law of large numbers for the martingale

$$
\int_{0}^{t} \sigma\left(z_{s}\right) d B_{s}+\int_{0}^{t} \int_{R^{d}} \gamma\left(z_{s}, y\right) \tilde{N}(d s, d y)
$$

is applicable and therefore

$$
\lim _{t \rightarrow \infty} \frac{1}{t} X_{i}(t)=\lim _{t \rightarrow \infty} \frac{1}{t} \int_{0}^{t} a_{i}\left(z_{s}\right) d s=\sum_{z^{\prime} \in D} a_{i}\left(z^{\prime}\right) \mu\left(z^{\prime}\right)=r_{i}
$$

$P$ a.e. Consequently, for large $t, X_{i}(t)$ is of order $t r_{i}$. If $r_{i}>r_{j}$, for $j \neq i$, then provided that $\pi_{i}(0)>0$ and the portfolio is not changed, it follows that

$$
\pi_{i}(t)=\frac{\pi_{i}(0) e^{X_{i}(t)}}{\sum_{j} \pi_{j}(0) e^{X_{j}(t)}} \rightarrow 1
$$

$P$ a.e. as $t \rightarrow \infty$, while $\pi_{j}(t) \rightarrow 0$ for $j \neq i$ as $t \rightarrow \infty P$ a.e.

In other words, assuming that the $r_{i}$ are not the same for $i=1,2, \ldots, d$, the process $\pi(t)$ in the limit converges to the boundary of $S$, provided that $\pi(0)>0$. As a consequence,

$$
\liminf _{t \rightarrow \infty} \frac{1}{t} E\left[\ln W_{t}\right]=\max _{i=1,2, \ldots, d} r_{i}
$$


This is the value of the wealth process that can be guaranteed over the long run. It may happen however that it is more profitable to change the portfolio regularly than just wait for the guaranteed value.

The portfolios from the boundary of $S$ are unacceptable from a risk sensitivity point of view. To eliminate risk, usually a portfolio is diversified. Therefore, in the paper an obligatory diversification is required.

A growth optimal portfolio is an important kind of portfolios studied in particular in the book [11] (see also references therein). In this paper a growth optimal portfolio with fixed proportional cost plus a cost proportional to the volume of transaction is considered. A model with only fixed proportional transaction $\operatorname{costs}(\kappa>0, c \equiv 0)$ was studied by Morton and Pliska in [10]. Long term growth with only proportional transaction costs was considered in [1]. A simple one asset Black Scholes model with fixed proportional costs plus proportional transaction costs was considered in [5], and the control was restricted to a diversification boundary and the choice of a new portfolio when this boundary was reached. This result has been generalized successively in [17] and [18], where a multidimensional version has been considered using quasi variational inequalities. In [15], general discrete and continuous time models with an obligatory diversification were studied. For a continuous time model, a certain transaction delay was introduced, which played an important role in the proofs. This paper generalizes [15] in various directions. A more specific asset growth model based on Lévy noise is considered. As in [15], the vanishing discount approach is used. To obtain continuity results a probabilistic version of penalty method is used. The main result, existence of the smooth solutions to the ergodic Bellman equation is obtained by the continuity properties of the transaction operator, which are shown in the paper, and finiteness of the space $D$. The existence of the smooth solutions to the Bellman equation is a fundamental result needed for the construction of nearly optimal portfolios (see [8]). This paper also generalizes [17] and [18] since more general asset prices model (including a Poisson term, and the dependence on asset prices model) is considered. Furthermore obligatory diversification which seems to be a rational assumption is imposed. The proofs in this paper are based on mainly probabilistic methods. A preliminary version of the paper, based on the time discretization instead of the penalty method has been presented at CDC08 (see [3] for a short version of the presentation).

\section{Properties of the Process $\pi$}

In this section we prove various properties of the uncontrolled process $(\pi(t))$, which will appear crucial to solve our optimization problem (7). We first show the form the transition operator $\Pi_{t}^{e}$ of the Markov process $\left(\pi(t), z_{t}, W_{t}\right)$ and its continuity in variation norm. Then we prove the finiteness of the exponential moment of the first exit time of $(\pi(t))$ from the set $S_{\delta}^{0}$, which corresponds to an obligatory moment of portfolio diversification. Finally, we show continuity properties of the stopped (at the first exit time from $S_{\delta}^{0}$ ) transition operator. 
Since $\left(z_{t}, t \geq 0\right)$ is a finite state continuous time, time homogeneous Markov process, its evolution can be described in the following form:

$$
\begin{aligned}
& \varsigma_{1}=\inf \left\{s \geq 0: z_{s} \neq z_{0}\right\}, \\
& \varsigma_{n+1}=\inf \left\{s \geq 0: z_{s+\varsigma_{n}} \neq z_{\varsigma_{n}}\right\}
\end{aligned}
$$

and for $z_{0}=z$

$$
\begin{aligned}
& P_{z}\left[\varsigma_{1} \leq t\right]=\int_{0}^{t} n(z, s) d s, \\
& P_{z}\left[P_{z_{\varsigma_{n}}}\left[\varsigma_{n+1} \leq t\right]\right]=E_{z}\left[\int_{0}^{t} n\left(z_{\varsigma_{n}}, s\right) d s\right], \\
& P_{z}\left[z\left(\varsigma_{1}\right)=z^{\prime}\right]=P\left(z, z^{\prime}\right) .
\end{aligned}
$$

We shall assume that $n(z, s)>0$ for $z \in D$ and $s>0$ and the matrix $P\left(z, z^{\prime}\right)$ is ergodic i.e. from one state we can enter the other with probability one (all states are communicative). We denote by $P_{t}(z, \cdot)$ the semigroup of transition operators corresponding to the Markov process $\left(z_{t}, t \geq 0\right)$. It is assumed further that

(A) $\sigma(z) \sigma(z)^{T}$ is uniformly elliptic, i.e. there is $\epsilon>0$ such that for $b \in R^{d}, z \in D$,

$$
b^{T} \sigma(z) \sigma(z)^{T} b \geq \epsilon b^{T} b .
$$

We have

Lemma 2 Under (A) the solution to (2) with the initial condition $X(0)=x$ has a continuous density $p_{t}$ for each fixed $z_{t}=z$ with respect to the Lebesgue measure $l^{d}$ at time $t>0$ i.e. for a Borel set $A \subset R^{d}$

$$
P_{x z}\left[X^{z}(t) \in A\right]=\int_{A} p_{t}^{z}\left(x, x^{\prime}\right) l^{d}\left(d x^{\prime}\right)
$$

where $X^{z}(t)$ is a solution to (2) with $z_{t} \equiv z$ and $p_{t}^{z}\left(x, x^{\prime}\right)$ is a continuous function of $x$ and $x^{\prime}$. Furthermore, given (A) for Borel set $B \subset R^{d}$ and $z^{\prime} \in D$,

$$
P_{x, z}\left[X(t) \in B, z_{t}=z^{\prime}\right]=\int_{B} p_{t}\left(x, x^{\prime}, z, z^{\prime}\right) l^{d}\left(d x^{\prime}\right)
$$

where

$$
\begin{aligned}
& p_{t}\left(x, x^{\prime}, z, z^{\prime}\right) \\
& =1_{z=z^{\prime}}\left(1-\int_{0}^{t} n(z, s) d s\right) p_{t}^{z}\left(x, x^{\prime}\right) \\
& \quad+\sum_{k=1}^{\infty} \sum_{z^{1} \in D} \sum_{z^{2} \in D} \ldots \sum_{z^{k-1} \in D} \int_{0}^{t} n\left(z, s_{1}\right) \int_{R^{d}} p_{s_{1}}^{z}\left(x, x_{1}\right) P\left(z, z^{1}\right)
\end{aligned}
$$




$$
\begin{aligned}
& \times \int_{0}^{t-s_{1}} n\left(z^{1}, s_{2}\right) \int_{R^{d}} p_{s_{2}}^{z^{1}}\left(x_{1}, x_{2}\right) P\left(z^{1}, z^{2}\right) \cdots \int_{0}^{t-s_{1}-\cdots-s_{k-1}} n\left(z^{k-1}, s_{k}\right) \\
& \times \int_{R^{d}} p_{s_{k}}^{z^{k-1}}\left(x_{k-1}, x_{k}\right) P\left(z^{k-1}, z^{\prime}\right) p_{t-s_{1}-\cdots-s_{k}}^{z^{\prime}}\left(x_{k}, y\right) \\
& \times \int_{t-s_{1}-\cdots-s_{k}}^{t} n\left(z^{\prime}, u\right) d u l\left(d x_{k}\right) d s_{k} l\left(d x_{k-1}\right) d s_{k-1} \ldots l\left(d x_{1}\right) d s_{1}
\end{aligned}
$$

Proof By (A) the solution to the equation $d D_{1}^{z}(t)=a(z) d t+\sigma(z) d B_{t}$ has a density $d_{t}^{z}(x, y)$ with respect to the Lebesgue measure $l^{d}$ and denoting by $F^{z}$ the distribution of $D_{2}^{z}(t)=\int_{0}^{t} \int_{R^{d}} \gamma(z, y) \tilde{N}(d s, d y)$ we have

$$
\begin{aligned}
E\left[f\left(X^{z}(t)\right)\right] & =E\left[f\left(D_{1}^{z}(t)+D_{2}^{z}(t)\right)\right]=\int_{R^{d}} f\left(D_{1}^{z}(t)+y^{\prime}\right) F^{z}\left(d y^{\prime}\right) \\
& =\int_{R^{d}} \int_{R^{d}} f\left(y+y^{\prime}\right) F^{z}\left(d y^{\prime}\right) d_{t}^{z}(x, y) l^{d}(d y) \\
& =\int_{R^{d}} \int_{R^{d}} f(y) d_{t}^{z}\left(x, y-y^{\prime}\right) F^{z}\left(d y^{\prime}\right) l^{d}(d y) .
\end{aligned}
$$

Consequently $p_{t}^{z}\left(x, x^{\prime}\right)=\int_{R^{d}} d_{t}^{z}\left(x, x^{\prime}-y^{\prime}\right) F^{z}\left(d y^{\prime}\right)$ from which (12) and the first part of Lemma follows. By direct calculation we obtain (13) and (14).

The following continuity property is crucial in further investigations:

Proposition 1 Under $(A)$, the operator $\Pi_{t}^{e}$ is continuous in variation norm for $(\pi, z, W) \in S_{\delta} \times D \times(0, \infty)$-i.e., for $\left(\pi_{(n)}, z, W_{(n)}\right) \rightarrow(\pi, z, W) \in S_{\delta} \times D \times$ $(0, \infty),\left(\pi_{(n)}, n \geq 1\right)$ is a sequence in $S_{\delta}$, and $\left(W_{(n)}, n \geq 1\right)$ a sequence in $(0, \infty)$ with $W>0$ it follows that

$$
\sup _{A \in B(S \times D \times(0, \infty))}\left|\Pi_{t}^{e}\left(\pi_{(n)}, z, W_{(n)}, A\right)-\Pi_{t}^{e}(\pi, z, W, A)\right| \rightarrow 0
$$

as $n \rightarrow \infty$, with $B(S \times D \times(0, \infty))$ denoting for the family of Borel subsets of $S \times D \times(0, \infty)$. In particular,

$$
\sup _{A \in B(S \times D)}\left|\Pi_{t}\left(\pi_{(n)}, z, A\right)-\Pi_{t}(\pi, z, A)\right| \rightarrow 0 .
$$

Proof Note that

$$
\Pi_{t}^{e}(\pi, z, W, A)=E_{\pi z}\left[1_{A}\left(\pi_{1}(t), \ldots, \pi_{d-1}(t), 1-\sum_{i=1}^{d-1} \pi_{i}(t), z_{t}, W \pi \cdot e^{X(t)}\right)\right]
$$


so the density of $\left(\pi_{1}(t), \ldots, \pi_{d-1}(t)\right)$ is used. Consider the following transformations of $R^{d}$ :

$$
\exp \left[\begin{array}{c}
x_{1} \\
x_{2} \\
\vdots \\
x_{d}
\end{array}\right]=\left[\begin{array}{c}
e^{x_{1}} \\
e^{x_{2}} \\
\vdots \\
e^{x_{d}}
\end{array}\right]
$$

for $\pi=\left(\pi_{1}, \pi_{2}, \ldots, \pi_{d}\right) \in S$,

$$
D_{\pi}\left[\begin{array}{c}
x_{1} \\
x_{2} \\
\vdots \\
x_{d}
\end{array}\right]=\left[\begin{array}{c}
\pi_{1} x_{1} \\
\pi_{2} x_{2} \\
\vdots \\
\pi_{d} x_{d}
\end{array}\right]
$$

and for $\left(x_{1}, x_{2}, \ldots, x_{d}\right) \in[0, \infty)^{d} \backslash\{(0, \ldots, 0)\}$,

$$
G\left[\begin{array}{c}
x_{1} \\
x_{2} \\
\cdots \\
x_{d-1} \\
x_{d}
\end{array}\right]=\left[\begin{array}{c}
x_{1} \\
\sum x_{i} \\
\sum x_{i} \\
\cdots \\
x_{d-1} \\
\sum x_{i} \\
\sum x_{i}
\end{array}\right] .
$$

The transformation $G D_{\pi}$ exp transforms $X(t)=\left(X_{1}(t), \ldots, X_{d}(t)\right)^{T}$ into

$$
\left(\pi_{1}(t), \pi_{2}(t), \ldots, \pi_{d-1}(t), \sum_{i=1}^{d} \pi_{i} e^{X_{i}(t)}\right)^{T}
$$

and the determinant of the inverse of the Jacobian is of the form

$$
\frac{1}{y_{1} y_{2} \ldots y_{d}} \frac{1}{\left(1-\sum_{i=1}^{d-1} y_{i}\right)} \text {. }
$$

Consequently, the density $f^{\pi, z}\left(y_{1}, \ldots, y_{d}, z^{\prime}\right)$ of

$$
\left(\pi_{1}(t), \pi_{2}(t), \ldots, \pi_{d-1}(t), \sum_{i=1}^{d} \pi_{i} e^{X_{i}(t)}, z(t)\right)
$$

is of the form (assuming that $z(0)=z$ and $z_{t}=z^{\prime}$ )

$$
\frac{p_{t}\left(0, \ldots, 0, \ln \frac{1}{\pi_{1}} y_{1} y_{d}, \ldots, \ln \frac{1}{\pi_{d-1}} y_{d-1} y_{d}, \ln \frac{1}{\pi_{d}}\left(1-\sum_{i=1}^{d-1} y_{i}\right) y_{d}, z, z^{\prime}\right)}{y_{1} y_{2} \ldots y_{d}\left(1-\sum_{i=1}^{d-1} y_{i}\right)} .
$$

By the Scheffé Theorem [19], pointwise convergence of transition densities implies convergence in $L^{1}$. Consequently, whenever $\pi_{(n)} \rightarrow \pi \in S_{\delta} W_{(n)} \rightarrow W>0$ it fol- 
lows, by continuity of $p_{t}$, that

$$
f^{\pi_{(n)}, z}\left(y_{1}, \ldots, y_{d-1}, \frac{y_{d}}{W_{(n)}}, z^{\prime}\right) \rightarrow f^{\pi, z}\left(y_{1}, \ldots, y_{d-1}, \frac{y_{d}}{W}, z^{\prime}\right)
$$

pointwise and the convergence is also in $L^{1}$. Therefore for any Borel measurable bounded function $h$ we have with $S^{r}=\left\{\left(v_{1}, \ldots, v_{d-1}\right), v_{i} \geq 0, \sum_{i=1}^{d-1} v_{i} \leq 1\right\}$

$$
\begin{aligned}
\Pi_{t}^{e}\left(\pi_{(n)}, z, W_{(n)}, h\right)= & E_{\pi_{(n)}, z, W_{(n)}}\left[h\left(\pi(t), z_{t}, W_{t}\right)\right] \\
= & \sum_{z^{\prime} \in D} \int_{S^{r} \times R^{+}} h\left(y_{1}, \ldots, y_{d-1}, 1-\sum_{i=1}^{d} y_{i}, z^{\prime}, W_{(n)} y_{d}\right) \\
& \times f^{\pi_{(n)}, z}\left(y_{1}, \ldots, y_{d}, z^{\prime}\right) l^{d}\left(y_{1}, \ldots, y_{d}\right) \\
= & \sum_{z^{\prime} \in D} \int_{S^{r} \times R^{+}} h\left(y_{1}, \ldots, y_{d-1}, 1-\sum_{i=1}^{d} y_{i}, z^{\prime}, y_{d}\right) \\
& \times \frac{1}{W_{(n)}} f^{\pi_{(n)}, z}\left(y_{1}, \ldots, y_{d-1}, \frac{y_{d}}{W_{(n)}}, z^{\prime}\right) \\
& \times l^{d}\left(y_{1}, \ldots, y_{d}\right) \rightarrow \Pi_{t}^{e}(\pi, z, W, h)
\end{aligned}
$$

from which (15) follows. Convergence (16) follows trivially from (15).

Let

$$
T^{\delta^{0}}=\inf \left\{s \geq 0: \pi(s) \in S \backslash S_{\delta}^{0}\right\}
$$

Remark 2 It is clear in view of Remark 1 that if the $r_{i}$ are not the same for all $i$, then $T^{\delta^{0}}<\infty, P$ a.e. The fact that $E_{\pi z}\left\{T^{\delta^{0}}\right\}<\infty$ follows mainly from the nondegeneracy of the diffusion term in the equation for $(\pi(t))$.

In what follows sufficient conditions for finiteness of the expected value of $T^{\delta^{0}}$ and higher moments are given.

Applying Ito's formula to the function $f_{i}^{\pi}\left(x_{1}, \ldots, x_{d}\right)=\left(\pi_{i} e^{x_{i}}\right) /\left(\sum \pi_{j} e^{x_{j}}\right)$, a stochastic differential equation is obtained for $\pi_{i}(t)$

$$
\begin{aligned}
d \pi_{i}(t)= & \tilde{a}_{i}\left(z_{t}, \pi\left(t^{-}\right)\right) d t+\tilde{\sigma}_{i}\left(z_{t}, \pi\left(t^{-}\right)\right) d B_{t} \\
& +\sum_{k=1}^{d} \int_{R} \tilde{\gamma}_{i k}\left(X\left(t^{-}\right), z_{t}, \pi\left(t^{-}\right), y_{k}\right) N_{k}\left(d t, d y_{k}\right)
\end{aligned}
$$

with

$$
\begin{aligned}
\tilde{a}_{i}\left(z_{t}, \pi\left(t^{-}\right)\right)= & \sum_{j=1}^{d} \pi_{i}\left(t^{-}\right) \tilde{\pi}_{j}^{i}\left(t^{-}\right)\left(a_{j}\left(z_{t}\right)-\sum_{k=1}^{d} \int_{R} \gamma_{i k}\left(z_{t}, y_{k}\right) v_{k}\left(d y_{k}\right)\right) \\
& +\sum_{k, j \neq i} \pi_{i}\left(t^{-}\right) \pi_{k}\left(t^{-}\right) \pi_{j}\left(t^{-}\right)\left(\sigma\left(z_{t}\right) \sigma\left(z_{t}\right)^{T}\right)_{k j}
\end{aligned}
$$




$$
\begin{gathered}
+\sum_{k \neq i} \pi_{k}\left(t^{-}\right) \pi_{i}\left(t^{-}\right)\left(2 \pi_{i}\left(t^{-}\right)-1\right)\left(\sigma\left(z_{t}\right) \sigma\left(z_{t}\right)^{T}\right)_{k i} \\
+\frac{1}{2}\left(2 \pi_{i}^{3}\left(t^{-}\right)-3 \pi_{i}^{2}\left(t^{-}\right)+\pi_{i}\left(t^{-}\right)\right)\left(\sigma\left(z_{t} \sigma\left(z_{t}\right)^{T}\right)_{i i},\right. \\
\tilde{\sigma}_{i}\left(z_{t}, \pi\left(t^{-}\right)\right)=\sum_{j=1}^{d} \pi_{i}\left(t^{-}\right) \tilde{\pi}_{j}^{i}\left(t^{-}\right) \sigma_{j}\left(z_{t}\right), \\
\tilde{\gamma}_{i k}\left(X\left(t^{-}\right), z_{t}, \pi\left(t^{-}\right), y_{k}\right)=f_{i}^{\pi}\left(X\left(t^{-}\right)+\gamma^{(k)}\left(z_{t}, y_{k}\right)\right)-f_{i}^{\pi}\left(X\left(t^{-}\right)\right)
\end{gathered}
$$

with $\tilde{\pi}_{i}^{i}(t)=1-\pi_{i}(t)$ and $\tilde{\pi}_{j}^{i}(t)=-\pi_{j}(t)$ for $i \neq j$, where $\gamma^{(k)}$ stands for the $k$-th column of the matrix $\gamma$ and $\left(\sigma(z) \sigma(z)^{T}\right)_{i j}$ is the $(i, j)$ entry of the matrix $\sigma(z) \sigma(z)^{T}$.

Notice that the matrix $\tilde{\sigma} \tilde{\sigma}^{T}$ is not uniformly elliptic. Clearly $\ell=(1,1, \ldots, 1) \tilde{\sigma}$ is a zero vector. The matrix $\tilde{\sigma} \tilde{\sigma}^{T}$ is however, uniformly elliptic on the subspace spanned by the vectors orthogonal to the vector $\ell=(1,1, \ldots, 1)^{T}$. In fact,

Lemma 3 Under $(A)$ the matrix $\tilde{\sigma}(z, \pi) \tilde{\sigma}(z, \pi)^{T}$ is uniformly elliptic for $b \in R^{d}$ such that $\sum_{i=1}^{d} b_{i}=0$ i.e. there is $\epsilon^{\prime}>0$ such that for $b \in R^{d}, \sum_{i=1}^{d} b_{i}=0, \pi \in S_{\delta}$, $z \in D$

$$
b^{T} \tilde{\sigma}(z, \pi) \tilde{\sigma}(z, \pi)^{T} b \geq \epsilon^{\prime} b^{T} b .
$$

Proof Assume that for a sequence of nonzero vectors $b^{n} \in R^{d}, \pi^{(n)} \in S_{\delta}$ and $z_{(n)} \in D$ it follows that $\left(b^{n}\right)^{T} \tilde{\sigma}\left(z_{(n)}, \pi^{(n)}\right) \tilde{\sigma}\left(z_{(n)}, \pi^{(n)}\right)^{T} b^{n} \rightarrow 0$ as $n \rightarrow \infty$ with $\sum_{i=1}^{d} b_{i}^{n}=0$. Since $b^{n} / \sqrt{\left(b^{n}\right)^{T} b^{n}}$ is on the unit sphere, $S_{\delta}$ and $D$ are compact, one can choose subsequences, for simplicity again denoted by $n$ such that $b^{n} / \sqrt{\left(b^{n}\right)^{T} b^{n}} \rightarrow \bar{b}$, where $\bar{b}$ is on the unit sphere, $\pi^{(n)} \rightarrow \pi \in S_{\delta}, z_{(n)} \rightarrow z \in D$. Letting $n \rightarrow \infty$

$$
\frac{\left(b^{n}\right)^{T}}{\sqrt{\left(b^{n}\right)^{T} b^{n}}} \tilde{\sigma}\left(z_{(n)}, \pi^{(n)}\right) \tilde{\sigma}\left(z_{(n)}, \pi^{(n)}\right)^{T} \frac{b^{n}}{\sqrt{\left(b^{n}\right)^{T} b^{n}}} \rightarrow 0
$$

by continuity, so it follows that $\bar{b}^{T} \tilde{\sigma}(z, \pi) \tilde{\sigma}(z, \pi)^{T} \bar{b}=0$. Since

$$
\bar{b}^{T} \tilde{\sigma}(z, \pi)=\sum_{i=1}^{d} \bar{b}_{i} \pi_{i} \sum_{j=1}^{d} \tilde{\pi}_{j}^{i} \sigma_{j}(z)=\sum_{j=1}^{d}\left(\sum_{i=1}^{d} \bar{b}_{i} \pi_{i} \tilde{\pi}_{j}^{i}\right) \sigma_{j}(z)
$$

by uniform ellipticity of $\sigma \sigma^{T}$, it follows that

$$
\sum_{i=1}^{d} \bar{b}_{i} \pi_{i} \tilde{\pi}_{j}^{i}=0
$$

for $j=1,2, \ldots, d$. The vectors $\left(\pi_{i} \tilde{\pi}_{j}^{i}\right)_{i=1,2, \ldots, d}$ are orthogonal to $\ell=(1,1, \ldots, 1)$ and since the matrix $\left(\pi_{i} \tilde{\pi}_{j}^{i}\right)$ is of rank $d-1$, vector $\ell$ is the unique (up to multiplica- 
tive constant) orthogonal vector. Consequently, $\bar{b}=c \ell$, with $c=1 / \sqrt{d}$, and that

$$
\frac{b_{i}^{n}}{\sqrt{\left(b^{n}\right)^{T} b^{n}}}-\frac{1}{\sqrt{d}} \rightarrow 0
$$

for $i=1,2, \ldots, d$.

Summing over $i$ in the last convergence, and using that $\sum_{i=1}^{d} b_{i}^{n}=0$, it follows that $-1 / \sqrt{d}=0$, a contradiction. Consequently, (25) is satisfied.

Corollary 1 Let $\mathcal{R}$ be the family of vectors $b \in R^{d}$ such that each of them has only one nonzero coordinate. Then under $(A)$ for $b \in \mathcal{R}, \pi \in S_{\delta}$ and $z \in D$ it follows

$$
b^{T} \tilde{\sigma}(z, \pi) \tilde{\sigma}(z, \pi)^{T} b \geq \frac{d-1}{d} \epsilon^{\prime} b^{T} b .
$$

Proof Each vector $b$ of $\mathcal{R}$ can be decomposed in a unique way into the sum $b^{0}+$ $b^{1}$ where $b^{0}$ is nonzero vector which is orthogonal to $\ell$ while $b^{1}=c \ell$. If the $k$-th coordinate of $b$ is nonzero say equal to $\bar{b}$, then $b^{0} k$-th coordinate of $b^{0}$ is $\bar{b}-c$, while the other coordinates are equal to $-c$. Since $b^{0}$ is orthogonal to $\ell$ it follows there is the equality $c=\frac{\bar{b}}{d}$. Therefore have

$$
\frac{b^{T} b}{\left(b^{0}\right)^{T} b^{0}}=\frac{\bar{b}^{2}}{\left(\bar{b}-\frac{\bar{b}}{d}\right)^{2}+(d-1)\left(\frac{\bar{b}}{d}\right)^{2}}=\frac{d}{d-1} .
$$

Since $\left(b^{1}\right)^{T} \tilde{\sigma}(z, \pi)=0$ using (28) we finally obtain

$$
b^{T} \tilde{\sigma}(z, \pi) \tilde{\sigma}(z, \pi)^{T} b \geq \epsilon^{\prime}\left(b^{0}\right)^{T} b^{0} \geq \frac{d-1}{d} \epsilon^{\prime} b^{T} b
$$

which completes the proof.

Lemma 4 Under $(A)$ the inequality

$$
\sup _{z \in D} \sup _{\pi \in S_{\delta}^{0}} E_{\pi z}\left[e^{\gamma T^{\delta^{0}}}\right]<\infty
$$

is satisfied a sufficiently small $\gamma$.

Proof Consider (following Corollary 3.7.2 of [7]) a Lyapunov function of the form $V(s, \eta)=e^{\theta s}\left[k-\eta_{i}^{2 n}\right]$ with positive $\theta, k$ and positive integer $n$ to be chosen later. By Ito's lemma it follows

$$
\begin{aligned}
& V\left(T^{\delta^{0}} \wedge t, \pi\left(T^{\delta^{0}} \wedge t\right)\right) \\
& =V(0, \pi(0))+\int_{0}^{T^{\delta^{0}} \wedge t} e^{\theta s}\left[\theta\left(k-\left(\pi_{i}\left(s^{-}\right)\right)^{2 n}\right)-2 n\left(\pi_{i}\left(s^{-}\right)\right)^{2 n-1} \tilde{a}_{i}\left(z_{s}, \pi\left(s^{-}\right)\right)\right. \\
& \left.\quad-n(2 n-1)\left(\tilde{\sigma}\left(z_{s}, \pi\left(s^{-}\right)\right) \tilde{\sigma}\left(z_{s}, \pi\left(s^{-}\right)\right)^{T}\right) i i\left(\pi_{i}\right)^{2 n-2}\left(s^{-}\right)\right] d s
\end{aligned}
$$




$$
\begin{aligned}
& +\sum_{k=1}^{d} \int_{0}^{T^{\delta^{0}} \wedge t} \int_{R}\left(V\left(s, \pi\left(s^{-}\right)+\tilde{\gamma}^{(k)}\left(X\left(s^{-}\right), z_{s}, \pi\left(s^{-}\right), y_{k}\right)\right)\right. \\
& \left.-V\left(s, \pi\left(s^{-}\right), y_{k}\right)\right) v_{k}\left(d y_{k}\right) d s \\
& -\int_{0}^{T^{\delta^{0}} \wedge t} e^{\theta s} 2 n\left(\pi_{i}\right)^{2 n-1}\left(s^{-}\right) \tilde{\sigma}_{i}\left(z_{s}, \pi\left(s^{-}\right)\right) d B_{s} \\
& +\sum_{k=1}^{d} \int_{0}^{T^{\delta^{0}} \wedge t} \int_{R}\left(V \left(s, \pi\left(s^{-}\right)+\tilde{\gamma}^{(k)}\left(X\left(s^{-}\right), z_{s}, \pi\left(s^{-}\right), y_{k}\right)\right.\right. \\
& \left.\left.-V\left(s, \pi\left(s^{-}\right)\right)\right)\right) \tilde{N}_{k}\left(d s, d y_{k}\right) .
\end{aligned}
$$

Now

$$
\begin{aligned}
& V\left(s, \pi\left(s^{-}\right)+\tilde{\gamma}^{(k)}\left(X\left(s^{-}\right), z_{s}, \pi\left(s^{-}\right), y_{k}\right)\right)-V\left(s, \pi\left(s^{-}\right), y_{k}\right) \\
& \left.\quad=-e^{\theta s}\left[\left(\pi_{i}\left(s^{-}\right)+\tilde{\gamma}_{i k}\left(X\left(s^{-}\right), z_{s}, \pi\left(s^{-}\right), y_{k}\right)\right)\right)^{2 n}-\left(\pi_{i}\left(s^{-}\right)\right)^{2 n}\right] .
\end{aligned}
$$

Taking expectation in (31), then using the martingale property of the integrals with respect to $d B$ and $\tilde{N}$ by (32) and Corollary 1 for $k>1$ there are a sufficiently large $n$ and small $\theta$ such that

$$
\begin{aligned}
\theta(k- & \left(\pi_{i}\left(s^{-}\right)^{n}\right)-2 n\left(\pi_{i}\left(s^{-}\right)\right)^{2 n-1}\|\tilde{a}\|-n(2 n-1) \frac{d-1}{d} \epsilon^{\prime}\left(\pi_{i}\left(s^{-}\right)\right)^{2 n-2} \\
& -e^{\theta s}\left[\left(\pi_{i}\left(s^{-}\right)+\tilde{\gamma}_{i k}\left(X\left(s^{-}\right), z_{s}, \pi\left(s^{-}\right), y_{k}\right)\right)^{2 n}-\left(\pi_{i}\left(s^{-}\right)\right)^{2 n}\right] \leq-\varpi<0
\end{aligned}
$$

whenever $\pi\left(s^{-}\right) \in S_{\delta}^{0}$, with a positive $\varpi$. Consequently

$$
E V\left(T^{\delta^{0}} \wedge t, \pi\left(T^{\delta^{0}} \wedge t\right)\right) \leq V(0, \pi(0))-E\left[\int_{0}^{T^{\delta^{0}} \wedge t} e^{\theta s} \varpi d s\right]
$$

and since $V \geq 0$

$$
E\left[\int_{0}^{T^{\delta^{0}} \wedge t} e^{\theta s} \varpi d s\right] \leq V(0, \pi(0))
$$

from which by the Fatou Lemma (30) follows.

In what follows the following property of the process $(\pi(t))$ is also used

Lemma 5 For any compact set $K \subset S^{0}, \epsilon>0, T>0$, there is a compact set $K^{\prime} \subset S^{0}$ such that

$$
\sup _{z \in D} \sup _{\pi \in K} P_{\pi z}\left[\pi(t) \notin K^{\prime} \quad \text { for some }[0, T]\right]<\epsilon \text {. }
$$

The proof of this lemma is based on Lemma 2 of [9]. 
Let

$$
\eta_{\pi, z, W}^{\epsilon}(t)=P_{\pi, z, W}\left\{\sup _{s \in[0, t]} \max \left\{\|\pi(s)-\pi\|,\|z(s)-z\|,\left|W_{s}-W\right|\right\} \geq \epsilon\right\}
$$

where $\|\cdot\|$ is the norm in $R^{d}$.

Lemma $6 \eta_{\pi, z, W}^{\epsilon}(t) \rightarrow 0$ uniformly for $\pi$ from compact subsets of $S^{0}, z \in D$ and for $W$ from compact subsets of $(0, \infty)$ as $t \rightarrow 0$.

Proof By the way the processes $(\pi(t))$ and $\left(W_{t}\right)$ are constructed it suffices to show that

$$
P_{z}\left[\sup _{s \in[0, t]}\|X(s)\| \geq \epsilon\right] \rightarrow 0
$$

as $t \rightarrow 0$. To prove the convergence (37) note that

$$
\begin{aligned}
P_{z}\left\{\sup _{s \in[0, t]}\|X(s)\| \geq \epsilon\right\} \leq & P_{z}\left[\sup _{s \in[0, t]} \int_{0}^{s}\|a(z(r))\| d r \geq \frac{\epsilon}{3}\right] \\
& +P_{z}\left[\sup _{s \in[0, t]}\left\|M_{1}(s)\right\| \geq \frac{\epsilon}{3}\right]+P_{z}\left[\sup _{s \in[0, t]}\left\|M_{2}(s)\right\| \geq \frac{\epsilon}{3}\right]
\end{aligned}
$$

where $M_{1}(t)=\int_{0}^{t} \sigma\left(z_{r}\right) d B_{r}, M_{2}(t)=\int_{0}^{t} \int_{R^{d}} \gamma\left(z_{r}, y\right) \tilde{N}(d r, d y)$. By the Thebyshev inequality, using the Doob's maximal inequality (see Theorem 1.3.8(iv) of [6]) we obtain (37).

The following continuity of the stopped semigroup will be important later

Lemma 7 For any continuous bounded function $F: S \times D \times(0, \infty) \rightarrow R$ and $t>0$ it follows that the mappings

$$
S \times D \times(0, \infty) \ni(\pi, z, W) \mapsto E_{\pi z W}\left[\chi_{t \leq T^{\delta^{0}}} F\left(\pi(t), z_{t}, W_{t}\right)\right]
$$

and

$$
S \times D \times(0, \infty) \ni(\pi, z, W) \mapsto E_{\pi z W}\left[F\left(\pi\left(t \wedge T^{\delta^{0}}\right), z_{t \wedge T^{\delta^{0}}}, W_{t \wedge T^{\delta^{0}}}\right)\right]
$$

are continuous.

Proof If $\pi \in S \backslash S_{\delta}^{0}$ the continuity follows directly from continuity of $F$. Assume that $\pi \in S_{\delta}^{0}$. Then for $s<t$ it follows that

$$
\begin{aligned}
E_{\pi z W}\left[\chi_{t \leq T^{\delta^{0}}} F\left(\pi(t), z_{t}, W_{t}\right)\right] \\
=E_{\pi z W}\left[\chi_{s \leq T^{\delta^{0}}} E_{\pi(s) z_{s} W_{s}}\left[\chi_{t-s \leq T^{\delta^{0}}} F\left(\pi(t-s), z_{t-s}, W_{t-s}\right)\right]\right] \\
=E_{\pi z W}\left[E_{\pi(s) z_{s} W_{s}}\left\{\chi_{t-s \leq T^{\delta^{0}}} F\left(\pi(t-s), z_{t-s}, W_{t-s}\right)\right]\right] \\
\quad-E_{\pi z W}\left[\chi_{s>T^{\delta^{0}}} E_{\pi(s) z_{s} W_{s}}\left[\chi_{t-s \leq T^{\delta^{0}}} F\left(\pi(t-s), z_{t-s}, W_{t-s}\right)\right]\right] \\
=a+b
\end{aligned}
$$


where the term a is continuous by Proposition 1 and letting $s \rightarrow 0$ the term b converges to 0 uniformly on compact subsets of $S_{\delta}^{0} \times D \times(0, \infty)$. Consequently we have continuity of (38). Similarly

$$
\begin{aligned}
E_{\pi z W}\left[\chi_{t>T^{\delta^{0}}} F\left(\pi\left(T^{\delta^{0}}\right), z_{T^{\delta^{0}}}, W_{T^{\delta^{0}}}\right)\right] \\
=E_{\pi z W}\left[\chi_{T^{\delta^{0}} \leq s} F\left(\pi\left(T^{\delta^{0}}\right), z_{T^{\delta^{0}}}, W_{T^{\delta^{0}}}\right)\right] \\
\quad+E_{\pi z W}\left[\chi_{T^{\delta^{0}}>s} E_{\pi(s) z_{s} W_{s}}\left[\chi_{t-s>T^{\delta^{0}}} F\left(\pi\left(T^{\delta^{0}}\right), z_{T^{\delta^{0}}}, W_{T^{\delta^{0}}}\right)\right]\right] \\
=E_{\pi z W}\left[\chi_{T^{\delta^{0}} \leq s} F\left(\pi\left(T^{\delta^{0}}\right), z_{T^{\delta^{0}}}, W_{T^{\delta^{0}}}\right)\right] \\
\quad+E_{\pi z W}\left[E_{\pi(s) z_{s} W_{s}}\left[\chi_{t-s>T^{\delta^{0}}} F\left(\pi\left(T^{\delta^{0}}\right), z_{T^{\delta^{0}}}, W_{T^{\delta^{0}}}\right)\right]\right] \\
\quad-E_{\pi z W}\left[\chi_{T^{\delta^{0}} \leq s} E_{\pi(s) z_{s} W_{s}}\left[\chi_{t-s>T^{\delta^{0}}} F\left(\pi\left(T^{\delta^{0}}\right), z_{T^{\delta^{0}}}, W_{T^{\delta^{0}}}\right)\right]\right] \\
=I+I I+I I I .
\end{aligned}
$$

Letting $s \rightarrow 0$ by Lemma 6 we have that I and III converge to 0 uniformly on compact subsets of $S_{\delta}^{0} \times D \times(0, \infty)$. The second term is a continuous function and by Proposition 1 converges to $E_{\pi z W}\left[\chi_{t>T^{\delta^{0}}} F\left(\pi\left(T^{\delta^{0}}\right), z_{T^{\delta^{0}}}, W_{T^{\delta^{0}}}\right)\right]$, uniformly on compact subsets of $S_{\delta}^{0} \times D \times(0, \infty)$, from which using continuity of (38) the continuity of (39) easily follows.

\section{Optimal Stopping-Penalty Method}

In this section we study a discounted optimal stopping problem using so called penalty method approach. As will be shown in Sects. 4 and 5 the solution to our optimization problem (7) can be reduced to a sequence of optimal stopping problems. Consequently in this section we show continuity of the optimal stopping value function and a form of optimal stopping times.

Consider now the following optimal discounted stopping problem

$$
w^{\alpha}(\pi, z, W)=\sup _{\tau} E_{\pi z}\left[e^{-\alpha \tau \wedge T^{\delta^{0}}}\left[\ln \left(W_{\tau \wedge T^{\delta^{0}}}\right)+F\left(\pi\left(\tau \wedge T^{\delta^{0}}\right), z_{\tau \wedge T^{\delta^{0}}}\right)\right]\right]
$$

with $\alpha>0$, and continuous and bounded function $F$. We shall approximate the function $w^{\alpha}(\pi, z, W)$ by the functions

$$
w_{N}^{\alpha}(\pi, z, W)=\sup _{\tau} E_{\pi z}\left[e^{-\alpha \tau \wedge T^{\delta^{0}}}\left[h_{N}\left(W_{\tau \wedge T^{\delta^{0}}}\right)+F\left(\pi\left(\tau \wedge T^{\delta^{0}}\right), z_{\tau \wedge T^{\delta^{0}}}\right)\right]\right]
$$

with $h_{N}(W)=\ln W$ whenever $|\ln W| \leq N$ and $h_{N}(W)=-N$ for $\ln W \leq-N$, $h_{N}(W)=N$ for $\ln W \geq N$. The following result is obtained

Lemma $8 w_{N}^{\alpha}(\pi, z, W) \rightarrow w^{\alpha}(\pi, z, W)$ uniformly in $\pi \in S, z \in D$ and $W$ from compact subsets of $(0, \infty)$. 
Proof Note that

$$
\begin{aligned}
-\sum_{i=1}^{d}\left|X_{i}\left(\tau \wedge T^{\delta^{0}}\right)\right| & \leq \sum_{i=1}^{d} \pi_{i} X_{i}\left(\tau \wedge T^{\delta^{0}}\right) \leq \ln \left(\pi \cdot e^{X\left(\tau \wedge T^{\delta^{0}}\right)}\right) \\
& =\ln \frac{W\left(\tau \wedge T^{\delta^{0}}\right)}{W} \leq \max _{i} X_{i}\left(\tau \wedge T^{\delta^{0}}\right) \\
& \leq \sum_{i=1}^{d}\left|X_{i}\left(\tau \wedge T^{\delta^{0}}\right)\right|
\end{aligned}
$$

and by Lemma $4, M_{1}(t)=\int_{0}^{t \wedge T^{\delta^{0}}} \sigma\left(z_{s}\right) d B_{s}$ and $M_{2}(t)=\int_{0}^{t \wedge T^{\delta^{0}}} \int_{R^{d}} \gamma\left(z_{s}, y\right) \tilde{N}(d s, d y)$ are uniformly square integrable martingales. Now

$$
\begin{aligned}
\left|w^{\alpha}(\pi, z, W)-w_{N}^{\alpha}(\pi, z, W)\right| & \leq \sup _{\tau} E_{\pi z}\left[e^{-\alpha \tau \wedge T^{\delta^{0}}} \chi\left|\ln W_{\tau \wedge T^{\delta}}\right| \geq N\left|\ln W_{\tau \wedge T^{\delta^{0}}}\right|\right] \\
& \leq \sup _{\tau}\left(P\left[\left|\ln W_{\tau \wedge T^{\delta^{0}}}\right| \geq N\right]\right)^{\frac{1}{2}}\left(E\left[\left(\ln W_{\tau \wedge T^{\delta^{0}}}\right)^{2}\right]\right)^{\frac{1}{2}} .
\end{aligned}
$$

By the form of (2) and Doob's maximal inequality (see Theorem 1.3.8 (iv) of [6]) applied to martingales $M_{1}$ and $M_{2}$ and Lemma 4 it follows that

$$
\begin{aligned}
E_{\pi z}\left[\sup _{s}\left|X_{i}\left(s \wedge T^{\delta^{0}}\right)\right|^{2}\right] \leq & 3 E_{\pi, z}\left[\left(\int_{0}^{T^{\delta^{0}}}\left|a_{i}\left(z_{t}\right)\right| d t\right)^{2}+\int_{0}^{T^{\delta^{0}}} \sum_{j=1}^{d} \sigma_{i j}^{2}\left(z_{t}\right) d t\right. \\
& \left.+\int_{0}^{T^{\delta^{0}}} \sum_{j=1}^{d} \int_{R} \gamma_{i j}^{2}\left(z_{t}, y\right) v_{j}(d y) d t\right]<\infty
\end{aligned}
$$

Therefore combining (42) with (43) and (44) the lemma is verified.

Consider now the following penalty equation for $\beta>0$

$$
\begin{aligned}
w_{N}^{\alpha \beta}(\pi, z, W)= & E_{\pi z}\left[\beta \int_{0}^{T^{\delta^{0}}} e^{-\alpha s}\left(h_{N}\left(W_{s}\right)+F\left(\pi(s), z_{s}\right)-w_{N}^{\alpha \beta}\left(\pi(s), z_{s}, W_{s}\right)\right)^{+} d s\right. \\
& \left.+e^{-\alpha T^{\delta^{0}}}\left[h_{N}\left(W_{T^{\delta}}\right)+F\left(\pi\left(T^{\delta^{0}}\right), z_{T^{\delta^{0}}}\right)\right]\right]
\end{aligned}
$$

where $(\cdot)^{+}$stays for a positive part of the term in bracket.

Proposition 2 For each $N>0$ there is a unique continuous bounded function $w_{N}^{\alpha \beta}$ satisfying (45). Furthermore denoting by $M_{\beta}$ the class of progressively measurable processes $\left(b_{s}\right)$ taking values in $[0, \beta]$ it follows that 


$$
\begin{aligned}
w_{N}^{\alpha \beta}(\pi, z, W)= & \sup _{b \in M_{\beta}} E_{\pi z W}\left[\int_{0}^{T^{\delta^{0}}} e^{-\int_{0}^{s}\left(\alpha+b_{u}\right) d u} b_{s}\left[h_{N}\left(W_{s}\right)+F\left(\pi(s), z_{s}\right)\right] d s\right. \\
& \left.+e^{-\int_{0}^{T^{\delta^{0}}}\left(\alpha+b_{u}\right) d u}\left[h_{N}\left(W_{T^{\delta^{0}}}\right)+F\left(\pi\left(T^{\delta^{0}}\right), z_{T^{\delta^{0}}}\right)\right]\right]
\end{aligned}
$$

and

$$
\begin{aligned}
w_{N}^{\alpha \beta}(\pi, z, W)= & \sup _{\tau} E_{\pi z W}\left[e ^ { - \alpha ( \tau \wedge T ^ { \delta ^ { 0 } } ) } \left(h_{N}\left(W_{\tau \wedge T^{\delta^{0}}}\right)+F\left(\pi\left(\tau \wedge T^{\delta^{0}}\right), z_{\tau \wedge T^{\delta 0}}\right)\right.\right. \\
& +\left(h_{N}\left(W_{\tau \wedge T^{\delta^{0}}}\right)+F\left(\pi\left(\tau \wedge T^{\delta^{0}}\right), z_{\tau \wedge T^{\delta^{0}}}\right)\right. \\
& \left.\left.-w_{N}^{\alpha \beta}\left(\pi\left(\tau \wedge T^{\delta^{0}}\right), z_{\tau \wedge T^{\delta^{0}}}, W_{\tau \wedge T^{\delta^{0}}}\right)\right)^{+}\right] .
\end{aligned}
$$

Proof Note first that by suitable version of Lemma 1 of [16] (45) is equivalent to

$$
\begin{aligned}
w_{N}^{\alpha \beta}(\pi, z, W)= & E_{\pi z}\left[\beta \int _ { 0 } ^ { T ^ { \delta ^ { 0 } } } e ^ { - ( \alpha + \beta ) s } \left[\left(h_{N}\left(W_{t}\right)+F\left(\pi(s), z_{s}\right)\right.\right.\right. \\
& \left.\left.-w_{N}^{\alpha \beta}\left(\pi(s), z_{s}, W_{s}\right)\right)^{+}+w_{N}^{\alpha \beta}\left(\pi(s), z_{s}, W_{s}\right)\right] d s \\
& \left.+e^{-(\alpha+\beta) T^{\delta^{0}}}\left[h_{N}\left(W_{T^{\delta^{0}}}\right)+F\left(\pi\left(T^{\delta^{0}}\right), z_{T^{\delta^{0}}}\right)\right]\right] .
\end{aligned}
$$

The mapping $\left.w \mapsto\left[h_{N}+F-w\right)^{+}+w\right]$ is Lipschitz with constant 1 . Therefore the right hand side of (48) is a contraction and by Lemma 7 it is a contraction in the space of continuous bounded functions, so that there is a unique bounded continuous solution to (45). For details see [16]. Using again Lemma 1 of [16] we obtain the following equivalent formula for (45) with $\left(b_{s}\right) \in M_{\beta}$

$$
\begin{aligned}
w_{N}^{\alpha \beta}(\pi, z, W)= & E_{\pi z W}\left[\int _ { 0 } ^ { T ^ { \delta ^ { 0 } } } e ^ { - \int _ { 0 } ^ { s } ( \alpha + b _ { u } ) d u } \left[\beta \left(h_{N}\left(W_{s}\right)\right.\right.\right. \\
& \left.\left.+F\left(\pi(s), z_{s}\right)-w_{N}^{\alpha \beta}\left(\pi(s), z_{s}, W_{s}\right)\right)^{+}+b_{s} w_{N}^{\alpha \beta}\left(\pi(s), z_{s}, W_{s}\right)\right] d s \\
& \left.+e^{-\int_{0}^{T^{\delta^{0}}}\left(\alpha+b_{u}\right) d u}\left[h_{N}\left(W_{T^{\delta^{0}}}\right)+F\left(\pi\left(T^{\delta^{0}}\right), z_{T^{\delta^{0}}}\right)\right]\right] .
\end{aligned}
$$

Note now that since $\left(b_{s}\right)$ is in $M_{\beta}$ we have $\beta\left(h_{N}+F-w_{N}^{\alpha \beta}\right)^{+}+b w_{N}^{\alpha \beta} \geq b\left(h_{N}+F\right)$ with equality for $b_{s}=\beta$ whenever $h_{N}\left(W_{s}\right)+F\left(\pi(s), z_{s}\right) \geq w_{N}^{\alpha \beta}\left(\pi(s), z_{s}, W_{s}\right)$ and $b_{s}=0$ otherwise. Therefore we obtain (46) (see also Lemma 2 of [16]).

From the penalty equation (45) for any stopping time $\tau$ we have

$$
\begin{aligned}
w_{N}^{\alpha \beta}(\pi, z, W)= & E_{\pi z}\left[\beta \int _ { 0 } ^ { T ^ { \delta ^ { 0 } } \wedge \tau } e ^ { - \alpha s } \left(h_{N}\left(W_{s}\right)+F\left(\pi(s), z_{s}\right)\right.\right. \\
& \left.-w_{N}^{\alpha \beta}\left(\pi(s), z_{s}, W_{s}\right)\right)^{+} d s \\
& \left.+e^{-\alpha T^{\delta^{0}} \wedge \tau} w_{N}^{\alpha \beta}\left(\pi\left(T^{\delta^{0}} \wedge \tau\right), z_{T^{\delta} \wedge \tau}, W_{T^{\delta^{0}} \wedge \tau}\right)\right] .
\end{aligned}
$$


Note that $w_{N}^{\alpha \beta} \geq h_{N}+F+\left(h_{N}+F-w_{N}^{\alpha \beta}\right)^{+}$so that

$$
\begin{aligned}
w_{N}^{\alpha \beta}(\pi, z, W) \geq & E_{\pi z W}\left[e ^ { - \alpha ( \tau \wedge T ^ { \delta ^ { 0 } } ) } \left(h_{N}\left(W_{\tau \wedge T^{\delta^{0}}}\right)\right.\right. \\
& +F\left(\pi\left(\tau \wedge T^{\delta^{0}}\right), z_{\tau \wedge T^{\delta^{0}}}\right)+\left(h_{N}\left(W_{\tau \wedge T^{\delta^{0}}}\right)+F\left(\pi\left(\tau \wedge T^{\delta^{0}}\right), z_{\tau \wedge T^{\delta^{0}}}\right)\right. \\
& \left.\left.-w_{N}^{\alpha \beta}\left(\pi\left(\tau \wedge T^{\delta^{0}}\right), z_{\tau \wedge T^{\delta^{0}}}, W_{\tau \wedge T^{\delta^{0}}}\right)\right)^{+}\right] .
\end{aligned}
$$

Since for $\tau=\inf \left\{t \geq 0: h_{N}\left(W_{t}\right)+F\left(\pi(t), z_{t}\right) \geq w_{N}^{\alpha \beta}\left(\pi(t), z_{t}, W_{t}\right)\right\}$ we have equality in the last formula, we finally obtain (47) (compare also to the proof of Lemma 3 of [16]).

Now the use of the penalty method is concluded

Theorem 1 The following uniform convergences on compact subsets of $S^{0} \times D \times$ $(0, \infty)$

$$
w_{N}^{\alpha \beta} \rightarrow w_{N}^{\alpha}
$$

as $\beta \rightarrow \infty$ and

$$
w_{N}^{\alpha} \rightarrow w^{\alpha}
$$

as $N \rightarrow \infty$ hold, so that the function $w^{\alpha}$ is bounded continuous on $S^{0} \times D \times(0, \infty)$. Furthermore

$$
\hat{\tau}=\inf \left\{t \geq 0: w^{\alpha}\left(\pi(t), z_{t}, W_{t}\right)=\ln W_{t}+F\left(\pi(t), z_{t}\right)\right\}
$$

is an optimal stopping time for (40).

Proof Note that by the form of $X(t)$ (see also Theorem 6.7.2 of [2]) and Proposition 1 the operator $\Pi_{t}^{e}$ transforms the class $C_{0}\left(S^{0} \times D \times(0, \infty)\right)$ of bounded continuous functions vanishing at the boundary of $S^{0} \times D \times(0, \infty)$ into itself (the boundary of $S^{0} \times D \times(0, \infty)$ consists of the sets $\partial S \times D \times(0, \infty)$ and $\left.S^{0} \times D \times\{0, \infty\}\right)$. Consequently as in Lemma 4 of [16] the class of functions $\phi$ of the form

$$
\begin{aligned}
\phi(\pi, z, W)= & E_{\pi z W}\left[\int_{0}^{T^{\delta^{0}}} e^{\alpha s} \psi_{1}\left(\pi(s), z_{s}, W_{s}\right) d s\right. \\
& \left.+e^{-\alpha T^{\delta^{0}}} \psi_{2}\left(\pi\left(T^{\delta^{0}}\right), z_{T^{\delta^{0}}}, W_{T^{\delta^{0}}}\right)\right]
\end{aligned}
$$

where $\psi_{1}$ and $\psi_{2} \in C_{0}\left(S^{0} \times D \times(0, \infty)\right)$ is dense in $C_{0}\left(S^{0} \times D \times(0, \infty)\right)$. Using Proposition 2, as in [16], it follows that $w_{N}^{\alpha \beta} \rightarrow w_{N}^{\alpha}$ as $\beta \rightarrow \infty$, uniformly on compact 
subsets of $S^{0} \times D \times(0, \infty)$ and therefore $w_{N}^{\alpha}$ is continuous on $S^{0} \times D \times(0, \infty)$. The convergence (50) follows directly from Lemma 8.

To show (51) consider the following stopping times for $\epsilon$ and $\epsilon^{\prime}>0$

$$
\begin{aligned}
& \hat{\tau}_{N}\left(\epsilon^{\prime}\right)=\inf \left\{t \geq 0: w_{N}^{\alpha}\left(\pi(t), z_{t}, W_{t}\right) \leq h_{N}\left(W_{t}\right)+F\left(\pi(t), z_{t}\right)+\epsilon^{\prime}\right\}, \\
& \hat{\tau}_{N}^{\beta}=\inf \left\{t \geq 0: w_{N}^{\alpha \beta}\left(\pi(t), z_{t}, W_{t}\right) \leq h_{N}\left(W_{t}\right)+F\left(\pi(t), z_{t}\right)\right\}, \\
& \hat{\tau}(\epsilon)=\inf \left\{t \geq 0: w^{\alpha}\left(\pi(t), z_{t}, W_{t}\right) \leq \ln W_{t}+F\left(\pi(t), z_{t}\right)+\epsilon\right\} .
\end{aligned}
$$

By the form of (45) we have that

$$
\begin{aligned}
& w_{N}^{\alpha \beta}(\pi, z, W)=E_{\pi z W}\left[e ^ { - \alpha ( \hat { \tau } ( \epsilon ) \wedge \hat { \tau } _ { N } ( \epsilon ^ { \prime } ) \wedge \hat { \tau } _ { N } ^ { \beta } ) } w _ { N } ^ { \alpha \beta } \left(\pi\left(\hat{\tau}(\epsilon) \wedge \hat{\tau}_{N}\left(\epsilon^{\prime}\right) \wedge \hat{\tau}_{N}^{\beta}\right),\right.\right.
\end{aligned}
$$

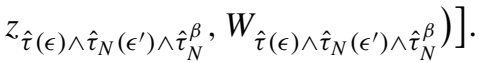

Since $w_{N}^{\alpha \beta} \rightarrow w_{N}^{\alpha}$ as $\beta \rightarrow \infty$, uniformly on compact subsets of $S^{0} \times D \times(0, \infty)$ we clearly have that $P\left\{\hat{\tau}_{N}^{\beta} \geq \hat{\tau}_{N}\left(\epsilon^{\prime}\right)\right\} \rightarrow 1$ as $\beta \rightarrow \infty$. Therefore letting $\beta \rightarrow \infty$ in the last equation we obtain

$$
\begin{gathered}
w_{N}^{\alpha}(\pi, z, W)=E_{\pi z W}\left[e ^ { - \alpha ( \hat { \tau } ( \epsilon ) \wedge \hat { \tau } _ { N } ( \epsilon ^ { \prime } ) ) } w _ { N } ^ { \alpha } \left(\pi\left(\hat{\tau}(\epsilon) \wedge \hat{\tau}_{N}\left(\epsilon^{\prime}\right)\right),\right.\right. \\
z_{\hat{\tau}(\epsilon) \wedge \hat{\tau}_{N}\left(\epsilon^{\prime}\right)}, W_{\left.\hat{\tau}(\epsilon) \wedge \hat{\tau}_{N}\left(\epsilon^{\prime}\right)\right]} .
\end{gathered}
$$

Letting now $\epsilon^{\prime} \rightarrow 0$, by quasi leftcontinuity of the processes $(\pi(t)),\left(z_{t}\right)$ and $\left(W_{t}\right)$, since $\hat{\tau}_{N}\left(\epsilon^{\prime}\right) \rightarrow \hat{\tau}_{N}(0)$ we obtain

$$
\begin{gathered}
w_{N}^{\alpha}(\pi, z, W)=E_{\pi z W}\left[e ^ { - \alpha ( \hat { \tau } ( \epsilon ) \wedge \hat { \tau } _ { N } ( 0 ) ) } w _ { N } ^ { \alpha } \left(\pi\left(\hat{\tau}(\epsilon) \wedge \hat{\tau}_{N}(0)\right),\right.\right. \\
\left.\left.z_{\hat{\tau}(\epsilon) \wedge \hat{\tau}_{N}(0)}, W_{\hat{\tau}(\epsilon) \wedge \hat{\tau}_{N}(0)}\right)\right] .
\end{gathered}
$$

Similarly as above $\lim _{N \rightarrow \infty} P\left[\hat{\tau}_{N}(0) \geq \hat{\tau}(\epsilon)\right]=1$ so that letting $N \rightarrow \infty$ in the last equation we now obtain

$$
w^{\alpha}(\pi, z, W)=E_{\pi z W}\left[e^{-\alpha \hat{\tau}(\epsilon)} w^{\alpha}\left(\pi(\hat{\tau}(\epsilon)), z_{\hat{\tau}(\epsilon)}, W_{\hat{\tau}(\epsilon)}\right)\right] .
$$

Letting now $\epsilon \rightarrow 0$, noting that $\hat{\tau}(\epsilon) \rightarrow \hat{\tau}$, by quasi leftcontinuity of $(\pi(t)),\left(z_{t}\right)$ and $\left(W_{t}\right)$ we finally obtain

$$
w^{\alpha}(\pi, z, W)=E_{\pi z W}\left[e^{-\alpha \hat{\tau}} w^{\alpha}\left(\pi(\hat{\tau}), z_{\hat{\tau}}, W_{\hat{\tau}}\right)\right],
$$

from which optimality of $\hat{\tau}$ follows. This completes the proof of the theorem.

\section{Discounted Growth Optimal Portfolio}

In this section we study so called discounted growth portfolio, which is used later as approximation to long run growth optimal portfolio. We prove continuity of the value function corresponding to optimal discounted growth portfolio and show that these functions are bounded with respect to the discount rate in the span norm. 
It is assumed in what follows that an impulsive strategy $V=\left(\tau_{n}, \pi^{n}\right)$ contains obligatory and nonobligatory transactions i.e., whenever $\pi(t)$ enters $S \backslash S_{\delta}^{0}$, it is required to make an obligatory transaction to $\pi^{\prime} \in S_{\delta^{\prime}}$ and when $\pi(t) \in S_{\delta}^{0}$ a transaction can be made but it is not required. Consider now the so-called discounted cost functional

$$
J_{\pi z}^{\alpha}(V)=E_{\pi z}\left[\sum_{i=1}^{\infty} e^{-\alpha \tau_{i}}\left[\ln \left(\pi\left(\tau_{i-1}\right) \cdot e^{X\left(\tau_{i}\right)-X\left(\tau_{i-1}\right)}\right)+\ln e\left(\pi^{-}\left(\tau_{i}\right), \pi\left(\tau_{i}\right)\right)\right]\right] .
$$

Let

$$
w^{\alpha}(\pi, z)=\sup _{V} J_{\pi z}^{\alpha}(V)
$$

Theorem 2 Under $(A), w^{\alpha}$ is a bounded function continuous on $S^{0}$ and is the unique solution to the following Bellman equation:

$$
w^{\alpha}(\pi, z)=\sup _{\tau} E_{\pi z}\left[e^{-\alpha \tau \wedge T^{\delta^{0}}}\left[\ln \left(\pi \cdot e^{X\left(\tau \wedge T^{\delta^{0}}\right)}\right)+M w^{\alpha}\left(\pi\left(\tau \wedge T^{\delta^{0}}\right), z\left(\tau \wedge T^{\delta^{0}}\right)\right)\right]\right]
$$

with

$$
M w(\pi, z)=\sup _{\pi^{\prime} \in S_{\delta^{\prime}}}\left[\ln e\left(\pi, \pi^{\prime}\right)+w\left(\pi^{\prime}, z\right)\right]
$$

Proof Let, for a continuous bounded function $w$ on $S_{\delta^{\prime}}$,

$$
\begin{aligned}
G^{\alpha} w(\pi, z)= & \sup _{\tau} E_{\pi z}\left[e ^ { - \alpha \tau \wedge T ^ { \delta ^ { 0 } } } \left[\ln \left(\pi \cdot e^{X\left(\tau \wedge T^{\delta^{0}}\right)}\right)\right.\right. \\
& \left.\left.+M w\left(\pi(\tau \wedge \sigma), z_{\tau \wedge T^{\delta^{0}}}\right)\right]\right] .
\end{aligned}
$$

By Theorem 1, the mapping

$$
\pi \rightarrow G^{\alpha} w(\pi, z)
$$

is continuous for $\pi \in S^{0}$. Let

$$
q^{\alpha}(\pi, z)=E_{\pi z}\left[\sum_{i=1}^{\infty} e^{-\alpha \tau_{i}}\left[\ln \left(\pi\left(\tau_{i-1}\right) \cdot e^{X\left(\tau_{i}\right)-X\left(\tau_{i-1}\right)}\right)+\ln e\left(\pi^{-}\left(\tau_{i}\right), \tilde{\pi}\right)\right]\right],
$$

where $\tau_{1}=T^{\delta^{0}}, \tau_{n+1}=\tau_{n}+T^{\delta^{0}} \circ \theta_{\tau_{n}}$ and $\pi_{n}=\tilde{\pi}$ with $\tilde{\pi} \in S_{\delta^{\prime}}$ fixed and $\theta_{t}$ denoted the Markov shift operator, i.e. $q^{\alpha}(\pi, z)$ is the value of the cost functional $J_{\pi z}^{\alpha}$ corresponding to only obligatory transactions to a fixed portfolio $\tilde{\pi} \in S_{\delta^{\prime}}$. Consider now the following sequence of functions

$$
\begin{aligned}
& q_{0}^{\alpha}(\pi, z)=q^{\alpha}(\pi, z), \\
& q_{1}^{\alpha}(\pi, z)=G^{\alpha} q_{0}^{\alpha}(\pi, z), \\
& q_{n}^{\alpha}(\pi, z)=G^{\alpha} q_{n-1}^{\alpha}(\pi, z) .
\end{aligned}
$$


Note that the value $q_{n}^{\alpha}(\pi, z)$ is the value of the cost functional with the strategy which consists of an optimal first $n$ transactions and then afterwards only obligatory transactions to $\tilde{\pi}$ (see Lemma V.2.2 in [12] for more details in the case of a general impulsive control). Therefore, it is clear that the sequence $q_{n}^{\alpha}(\pi, z)$ is increasing. Consequently there is a limit $\hat{q}^{\alpha}(\pi, z)$ and letting $n \rightarrow \infty$ in (58) it follows that

$$
\hat{q}^{\alpha}(\pi, z)=G^{\alpha} \hat{q}^{\alpha}(\pi, z) .
$$

Since for any bounded function $f$, the function $\pi \rightarrow M f(\pi, z)$ is continuous (by the continuity of $e\left(\pi, \pi^{\prime}\right)$ ), using Theorem 1 there is continuity of $\hat{q}^{\alpha}(\pi, z)$ for $\pi \in S^{0}$. The function $\hat{q}^{\alpha}$ is therefore a solution to (54) for $\pi \in S^{0}, z \in D$. From the form of $q_{n}(\alpha)$ it is clear that $\hat{q}^{\alpha}$ coincides with $w^{\alpha}$, which completes the proof.

Remark 3 In the proof of Theorem 2, a smoothing property of the operator $M$ is used. Alternatively, one could use the fact that due to a fixed proportional transaction cost it is not optimal to have too many transactions in a finite time interval. This method allows one to prove a version of Theorem 2 for processes $(z(t))$ taking values in a general (not necessarily finite) state space whose transition probabilities are continuous in variation norm. Furthermore notice that because of the cost functional (52) the process $\left(W_{t}\right)$ in the definition in $w^{\alpha}$ may be neglected since after each transaction we start with $W=1$, which is due to stationarity of the process $X(t)$ (see (2)). Consequently $w^{\alpha}(\pi, z)$ as a solution to $(54)$ corresponds to $w^{\alpha}(\pi, z, 1)$ defined in (40) with $F(\pi, z)$ equal to $\sup _{\pi^{\prime} \in S_{\delta^{\prime}}}\left[\ln e\left(\pi, \pi^{\prime}\right)+w^{\alpha}\left(\pi^{\prime}, z, 1\right)\right]$. A more general form of the process $(X(t))$ would result in the dependence of $w^{\alpha}$ on the current value of the process $(X(t))$.

The following property of $w^{\alpha}$ will be important later:

Corollary 2 For $\pi, \pi^{\prime} \in S_{\delta^{\prime}}^{0}$ and $z \in D$, it follows that

$$
\left|w^{\alpha}(\pi, z)-w^{\alpha}\left(\pi^{\prime}, z\right)\right| \leq\left|\ln e\left(\pi, \pi^{\prime}\right)\right|+\left|\ln e\left(\pi^{\prime}, \pi\right)\right|
$$

and for $\pi \in S, \pi^{\prime} \in S_{\delta^{\prime}}^{0}$,

$$
\ln e\left(\pi, \pi^{\prime}\right)+w^{\alpha}\left(\pi^{\prime}, z\right) \leq w^{\alpha}(\pi, z)=M w^{\alpha}(\pi, z) .
$$

Proof For $\pi, \pi^{\prime} \in S_{\delta}^{0}$,

$$
w^{\alpha}(\pi, z) \geq M w^{\alpha}(\pi, z) \geq \ln e\left(\pi, \pi^{\prime}\right)+w^{\alpha}\left(\pi^{\prime}, z\right),
$$

and

$$
w^{\alpha}\left(\pi^{\prime}, z\right) \geq \ln e\left(\pi^{\prime}, \pi\right)+w^{\alpha}(\pi, z),
$$

and (61) is immediate from the definition of $M$.

To study a long time growth optimal function $J(V)$ of the form (7), a uniform ergodicity of the Markov process $(z(t))$, shown below, is important. 
Lemma 9 For each $t>0$ there is $\Delta(t)<1$ such that

$$
\sup _{z, z^{\prime} \in D} \sup _{A \subset D}\left|P_{t}(z, A)-P_{t}\left(z^{\prime}, A\right)\right|=\Delta(t)<1
$$

Proof Since all states are communicative and we have a finite state space $D$, there is a positive integer $k$ such that all entries of the matrix $P^{k}$ are positive. Now since $n(z, s)>0$ for each $z \in D$ and $s>0$ with a positive probability we have exactly $k$ jumps of the process $(z(s))$ till time $t$ and therefore $P_{t}\left(z^{\prime}, z^{\prime \prime}\right)>0$ for $z^{\prime}, z^{\prime \prime} \in D$. Let $\inf _{z^{\prime}, z^{\prime \prime} \in D} P_{t}\left(z^{\prime}, z^{\prime \prime}\right)=\kappa$, then $P_{t}(z, A)-P_{t}\left(z^{\prime}, A\right) \leq 1-\kappa$ for any $z, z^{\prime} \in D$ and $A \subset D$, and the assertion of lemma holds for $\Delta(t)=1-\kappa$.

Let

$$
h^{\alpha}(\pi, z)=w^{\alpha}(\pi, z)-\inf _{\pi^{\prime} \in S, z^{\prime} \in D} w^{\alpha}\left(\pi^{\prime}, z^{\prime}\right) .
$$

Proposition 3 Under $(A)$ there are $\Delta<1$ and a constant $M$ independent of $\alpha$ such that

$$
\sup _{\pi \in S, z \in D} h^{\alpha}(\pi, z) \leq \frac{M}{1-\Delta} .
$$

Proof The Bellman equation (54) can be also written in the following equivalent form for every $N \geq 0$

$$
\begin{aligned}
w^{\alpha}(\pi, z)= & \sup _{\tau} E_{\pi z}\left[e ^ { - \alpha \tau \wedge T ^ { \delta ^ { 0 } } \wedge N } \left[\ln \pi \cdot e^{X\left(\tau \wedge T^{\delta^{0}} \wedge N\right)}\right.\right. \\
& +1_{\tau \wedge T^{\delta^{0}}<N} M w^{\alpha}\left(\pi\left(\tau \wedge T^{\delta^{0}}\right), z_{\tau \wedge T^{\delta^{0}}}\right) \\
& \left.\left.+1_{\tau \wedge T^{\delta^{0}} \geq N} w^{\alpha}\left(\pi(N), z_{N}\right)\right]\right] .
\end{aligned}
$$

Iterating (64), the following equalities are satisfied

$$
\begin{aligned}
w^{\alpha}(\pi, z)= & \sup _{V} E_{\pi z}\left[\sum _ { i = 1 } ^ { \infty } e ^ { - \alpha \tau _ { i } \wedge N } \left(\ln \pi\left(\tau_{i-1} \wedge N\right) \cdot e^{\left(X\left(\tau_{i} \wedge N\right)-X\left(\tau_{i-1} \wedge N\right)\right)}\right.\right. \\
& \left.+\ln e\left(\pi^{-}\left(\tau_{i}\right), \pi\left(\tau_{i}\right)\right) 1_{\tau_{i} \leq N}+e^{-\alpha N} w^{\alpha}\left(\pi(N), z_{N}\right)\right] .
\end{aligned}
$$

Fix $\bar{\pi} \in S_{\delta}^{0}$. It is claimed that there is a constant $C$ such that for any $\pi \in S, z \in D$

$$
w^{\alpha}(\pi, z) \leq C+w^{\alpha}(\bar{\pi}, z)
$$

Assume that starting from $(\pi, z)$, an optimal time $\hat{\tau} \wedge T^{\delta^{0}}(\pi)$ for the first transaction is determined and at this moment portfolio $\bar{\pi}$ is chosen. Starting from $(\bar{\pi}, z)$ make only obligatory transactions until $\hat{\tau} \wedge T^{\delta^{0}}(\pi)$, each time choosing portfolio $\bar{\pi}$ 
and at $\hat{\tau} \wedge T^{\delta^{0}}(\pi)$ choose again portfolio $\bar{\pi}$. By $T^{\delta^{0}}(\pi)$ above we denote the first exit time from $S_{\delta}^{0}$ by $(\pi(t))$ starting from $\pi$. Then, from (60) it follows

$$
\begin{aligned}
w^{\alpha}(\pi, z)-w^{\alpha}(\bar{\pi}, z) \leq & E_{\pi z}\left[e ^ { - \alpha \hat { \tau } \wedge T ^ { \delta ^ { 0 } } ( \pi ) } \left[\ln \pi \cdot e^{X\left(\hat{\tau} \wedge T^{\delta^{0}}(\pi)\right)}\right.\right. \\
& \left.\left.+2 K+w^{\alpha}\left(\bar{\pi}, z_{\hat{\tau} \wedge T^{\delta}(\pi)}\right)\right]\right]-\left(J_{\bar{\pi}, z}\left(\hat{\tau} \wedge T^{\delta^{0}}(\pi)\right)-K\right. \\
& \left.+E_{\bar{\pi}, z}\left[e^{-\alpha \hat{\tau} \wedge T^{\delta^{0}}(\pi)} w^{\alpha}\left(\bar{\pi}, z_{\hat{\tau} \wedge T^{\delta^{0}}(\pi)}\right)\right]\right) \\
\leq & C_{1}+3 K+C_{2}=C
\end{aligned}
$$

where $J_{\bar{\pi}, z}(\hat{\tau} \wedge \sigma(\pi))$ is a cost functional of the strategy consisting of obligatory transactions to $\bar{\pi}$ and stopped at $\hat{\tau} \wedge \sigma(\pi)$ which by Lemma 4 is bounded uniformly by $C_{2}$. Furthermore,

$$
K=\sup _{\pi \in S, \pi^{\prime} \in S_{\delta}}\left|\ln e\left(\pi, \pi^{\prime}\right)\right|,
$$

and

$$
\sup _{\pi \in S, z \in D} E_{\pi z}\left[e^{-\alpha \tau \wedge T^{\delta^{0}}}\left(\ln \pi e^{X\left(\tau \wedge T^{\delta^{0}}\right)}\right)\right] \leq C_{1},
$$

which verifies (66).

By (65), (66), (61), and (67),

$$
\begin{aligned}
& w^{\alpha}(\pi, z)-w^{\alpha}\left(\pi^{\prime}, z^{\prime}\right) \leq \sup _{V} J_{\pi z}^{\alpha N}(V)+C+e^{-\alpha N} E_{\pi z}\left[w^{\alpha}\left(\bar{\pi}, z_{N}\right)\right] \\
&-\left(\sup _{V} J_{\pi^{\prime}, z^{\prime}}^{\alpha N}(V)-K+e^{-\beta N} E_{\pi^{\prime}, z^{\prime}}\left[w^{\alpha}\left(\bar{\pi}, z_{N}\right)\right]\right) \\
& \leq M+e^{-\alpha N}\left(E_{\pi z}\left[w^{\alpha}\left(\bar{\pi}, z_{N}\right)\right]-E_{\pi z^{\prime}}\left[w^{\alpha}\left(\bar{\pi}, z_{N}\right)\right]\right) \\
&= M+e^{-\alpha N}\left[\sum_{z^{\prime \prime} \in D_{1}} w^{\alpha}\left(\bar{\pi}, z^{\prime}\right)\left(P_{N}\left(z, z^{\prime \prime}\right)-P_{N}\left(z^{\prime}, z^{\prime \prime}\right)\right)\right. \\
&\left.+\sum_{z^{\prime \prime} \notin D_{1}} w^{\beta}\left(\bar{\pi}, z^{\prime}\right)\left(P_{N}\left(z, z^{\prime \prime}\right)-P_{N}\left(z^{\prime}, z^{\prime \prime}\right)\right)\right] \\
& \leq M+\Delta\left(\sup _{z^{\prime}} w^{\alpha}\left(\bar{\pi}, z^{\prime}\right)-\inf _{z^{\prime}} w^{\alpha}\left(\bar{\pi}, z^{\prime}\right)\right)
\end{aligned}
$$

where $D_{1}=\left\{z^{\prime \prime} \in D: P_{N}\left(z, z^{\prime \prime}\right) \geq P_{N}\left(z^{\prime}, z^{\prime \prime}\right)\right\}$, from which (63) is obtained.

\section{Long View Growth Optimal Portfolio}

In this final section we prove the existence of solutions to the Bellman equation corresponding to long run growth optimal portfolio and show the form of optimal strategies. 
Now rewrite the Bellman equation (54) in terms of a bounded (by Proposition 3) function $h^{\alpha}$. Thus

$$
\begin{aligned}
h^{\alpha}(\pi, z)= & \sup _{\tau} E_{\pi z}\left[e ^ { - \alpha \tau \wedge T ^ { \delta ^ { 0 } } } \left[\ln \left(\pi \cdot e^{X\left(\tau \wedge T^{\delta^{0}}\right)}\right)\right.\right. \\
& +M h^{\alpha}\left(\pi(\tau \wedge \sigma), z_{\left.\left.\tau \wedge T^{\delta^{0}}\right)\right]}\right. \\
& \left.-\inf _{\pi^{\prime} \in S, z^{\prime} \in D} w^{\alpha}\left(\pi^{\prime}, z^{\prime}\right)\left(1-e^{-\alpha \tau \wedge T^{\delta^{0}}}\right)\right] .
\end{aligned}
$$

The main result of the paper can be formulated as follows:

Theorem 3 Under (A) there exist a constant $\lambda$ and a continuous bounded function $w$ such that

$$
\begin{aligned}
w(\pi, z)= & \sup _{\tau} E_{\pi z}\left[\ln \left(\pi \cdot e^{X\left(\tau \wedge T^{\delta^{0}}\right)}\right)-\lambda\left(\tau \wedge T^{\delta^{0}}\right)\right. \\
& \left.+M w\left(\pi\left(\tau \wedge T^{\delta^{0}}\right), z_{\tau \wedge T^{\delta^{0}}}\right)\right] .
\end{aligned}
$$

Moreover,

$$
\lambda=\sup _{V} J(V)
$$

i.e., $\lambda$ is the optimal value of the cost functional (7) and the strategy $\hat{V}=\left(\hat{\tau}_{n}, \hat{\pi}^{n}\right)$ such that

$$
\begin{aligned}
& \hat{\tau}(\pi)=\inf \left\{s \geq 0: w\left(\pi(s), z_{s}\right)=M w\left(\pi(s), z_{s}\right)\right\}, \\
& \hat{\tau_{1}}=\hat{\tau}(\pi(0)), \\
& \hat{\tau}_{n+1}=\hat{\tau}_{n}+\hat{\tau}\left(\pi\left(\hat{\tau}_{n}\right)\right) \circ \theta_{\hat{\tau}_{n}}
\end{aligned}
$$

and

$$
\hat{\pi}^{n}=\hat{\pi}\left(\pi^{-}\left(\hat{\tau}_{n}\right), z_{\hat{\tau}_{n}}\right)
$$

where $\hat{\pi}: S \times D \rightarrow S_{\delta^{\prime}}$ is a Borel function such that

$$
M w(\pi, z)=\ln e(\pi, \hat{\pi}(\pi, z))+w(\hat{\pi}(\pi, z), z)
$$

is optimal.

Proof Note that

$$
\inf _{\pi^{\prime} \in S, z^{\prime} \in D} \alpha w^{\alpha}\left(\pi^{\prime}, z^{\prime}\right)
$$

is bounded so that there is a constant $\lambda$ and a sequence $\alpha_{n} \downarrow 0$ such that

$$
\inf _{\pi^{\prime} \in S, z^{\prime} \in D} \alpha_{n} w^{\alpha_{n}}\left(\pi^{\prime}, z^{\prime}\right) \rightarrow \lambda
$$


as $n \rightarrow \infty$. Furthermore, by Lemma 4, taking into account that for $x \geq 0$ we have $0 \leq 1-e^{-x} \leq x$, using dominated convergence theorem we obtain

$$
E_{\pi z}\left[\frac{1}{\alpha_{n}}\left(1-e^{-\alpha_{n} \tau \wedge T^{\delta^{0}}}\right)\right] \rightarrow E_{\pi z}\left[\tau \wedge T^{\delta^{0}}\right]
$$

as $n \rightarrow \infty$, and the limit is uniform in $\tau, \pi$, and $z$. By Proposition 3, the functions $h^{\alpha}$ are bounded. Therefore, $M h^{\alpha}(\pi, z)$ is uniformly continuous in $\pi \in S$ (use the continuity of $e$ ). One can therefore choose a subsequence of $\alpha_{n}$, for simplicity again denoted by $\alpha_{n}$, such that

$$
M h^{\alpha_{n}}(\pi, z) \rightarrow h(\pi, z)
$$

uniformly, where $h(\pi, z)$ is a continuous function of $\pi$. Therefore, by (68), there is a continuous function $w$ such that

$$
\sup _{\pi \in S} \sup _{z \in D}\left|h^{\alpha_{n}}(\pi, z)-w(\pi, z)\right| \rightarrow 0
$$

as $n \rightarrow \infty$. From (73), it follows that

$$
M h^{\alpha_{n}}(\pi, z) \rightarrow M w(\pi, z)=h(\pi, z)
$$

uniformly in $\pi \in S, z \in D$.

Finally, $w$ is a solution to (69). By the proof of Theorem 1 the optimal stopping time $\hat{\tau}$ for (69) is of the form

$$
\hat{\tau}=\inf \left\{s \geq 0: \bar{w}\left(\pi(s), z_{s}, W_{s}\right)=\ln W_{s}+M w\left(\pi(s), z_{s}\right)\right\}
$$

where

$$
\begin{aligned}
\bar{w}(\pi, z, W)= & \sup _{\tau} E_{\pi z}\left[\ln \left(W \pi \cdot e^{X\left(\tau \wedge T^{\delta^{0}}\right)}\right)-\lambda\left(\tau \wedge T^{\delta^{0}}\right)\right. \\
& \left.+M w\left(\pi\left(\tau \wedge T^{\delta^{0}}\right), z_{\tau \wedge T^{\delta^{0}}}\right)\right] .
\end{aligned}
$$

Clearly $\bar{w}(\pi, z, W)=\ln W+w(\pi, z)$ and therefore the optimal stopping time $\hat{\tau}$ is of the form (71). Equality (70) can be justified in a standard way for impulsive control of Markov processes as Theorem V.2.1 of [12] (see also a remark in [13]), using suitable version of Lemma II.2.2 of [12]. For completeness we present here a full proof. For a given impulsive strategy $V=\left(\tau_{n}, \pi^{n}\right)$ consider the following notation: for $n=1,2, \ldots, \pi_{n}\left(\tau_{n}\right)=\pi^{n}, \pi_{n}\left(\tau_{n}+s\right)=\pi^{-}\left(\tau_{n}+s\right)$ for $s>0, W_{n}\left(\tau_{n}\right)=1$, and $W_{n}\left(\tau_{n}+s\right)=W_{\tau_{n}+s}^{-}=\pi^{n} \cdot e^{X\left(\tau_{n}+s\right)-X\left(\tau_{n}\right)}$ for $s>0$. It is clear that from (74)

$$
\bar{w}\left(\pi_{n-1}\left(\tau_{n}\right), z_{\tau_{n}}, W_{n-1}\left(\tau_{n}\right)\right) \geq \ln W_{n-1}\left(\tau_{n}\right)+\ln e\left(\pi_{n-1}\left(\tau_{n}\right), \pi^{n}\right)+\bar{w}\left(\pi^{n}, z_{\tau_{n}}, 1\right) .
$$

By the form (74) of the function $\bar{w}$ we have that

$$
Z_{n}(s)=\bar{w}\left(\pi_{n}\left(\tau_{n}+s\right), z_{\tau_{n}+s}, W_{n}\left(\tau_{n}+s\right)\right)-\lambda s
$$


is a $\mathcal{G}_{s}^{n}=\mathcal{F}_{\tau_{n}+s}$ supermartingale. For any stopping time $\tau \geq \tau_{n}$ we have $\left\{\tau-\tau_{n} \leq s\right\}=$ $\left\{\tau \leq \tau_{n}+s\right\} \in \mathcal{F}_{\tau_{n}+s}=\mathcal{G}_{s}^{n}$, which means that $\tau-\tau_{n}$ is a $\left(\mathcal{G}_{s}^{n}\right)$ stopping time. Therefore

$$
E\left[Z_{n}\left(\tau-\tau_{n}\right) \mid \mathcal{F}_{\tau_{n}}\right] \leq Z_{n}(0)=\bar{w}\left(\pi_{n}\left(\tau_{n}\right), z_{\tau_{n}}, W_{n}\left(\tau_{n}\right)\right) .
$$

Consequently for fixed $T>0$ we have (using also (75))

$$
\begin{aligned}
& E\left[Z_{n}\left(\tau_{n+1} \wedge T-\tau_{n}\right) \chi_{\tau_{n} \leq T} \mid \mathcal{F}_{\tau_{n}}\right] \\
& \quad \leq \chi_{\tau_{n} \leq T} Z_{n}(0) \\
& \quad \leq \chi_{\tau_{n} \leq T}\left(\bar{w}\left(\pi_{n-1}\left(\tau_{n}\right), z_{\tau_{n}}, W_{n-1}\left(\tau_{n}\right)\right)-\ln \left(W_{n-1}\left(\tau_{n}\right) e\left(\pi_{n-1}\left(\tau_{n}\right), \pi^{n}\right)\right) .\right.
\end{aligned}
$$

Therefore

$$
\begin{aligned}
E\left[\chi _ { \tau _ { n } \leq T } \left(\bar{w}\left(\pi_{n}\left(\tau_{n+1} \wedge T\right), z_{\tau_{n+1} \wedge T}, W_{n}\left(\tau_{n+1} \wedge T\right)\right)\right.\right. \\
\left.\left.\quad-\bar{w}\left(\pi_{n-1}\left(\tau_{n}\right), z_{\tau_{n}}, W_{n-1}\left(\tau_{n}\right)\right)+\ln \left(W_{n-1}\left(\tau_{n}\right) e\left(\pi_{n-1}\left(\tau_{n}\right), \pi^{n}\right)\right)\right)\right] \\
\leq E\left[\chi_{\tau_{n} \leq T} \lambda\left(\tau_{n+1} \wedge T-\tau_{n}\right)\right] .
\end{aligned}
$$

Summing up over $n$ the inequalities (78) we obtain the formula

$$
\begin{aligned}
& E\left[\overline { w } \left(\pi_{\zeta(T)-1}\left(\tau_{\zeta(T)} \wedge T\right), z_{\tau_{\zeta(T)} \wedge T}, W_{\zeta(T)-1}\left(\tau_{\zeta(T)} \wedge T\right)-\bar{w}(\pi, z, W)\right.\right. \\
& \left.\quad+\sum_{i=0}^{\zeta(T)-1} \ln \left(W_{i}\left(\tau_{i+1}\right) e\left(\pi_{i}\left(\tau_{i+1}\right), \pi^{i+1}\right)\right)\right] \leq \lambda T
\end{aligned}
$$

where $\zeta(T)=\inf \left\{n: \tau_{n} \geq T\right\}$. Note that for the strategy $\hat{V}$ defined in (71) and (72) we have equalities in (78)-(79). Dividing both sides of (79) by $T$ and letting $T \rightarrow \infty$ we obtain (70) and optimality of the strategy $\hat{V}$. This completes the proof of Theorem 3 .

Acknowledgement The authors thank the referee for pointing out a gap in the proof of Theorem 1 .

Open Access This article is distributed under the terms of the Creative Commons Attribution Noncommercial License which permits any noncommercial use, distribution, and reproduction in any medium, provided the original author(s) and source are credited.

\section{References}

1. Akian, M., Sulem, A., Taksar, M.I.: Dynamic optimization of long-term growth tate for a portfolio with transaction costs and logarithmic utility. Math. Finance 11, 153-188 (2001)

2. Applebaum, D.: Lévy Processes and Stochastic Calculus. Cambridge University Press, Cambridge (2004)

3. Duncan, T., Pasik-Duncan, B., Stettner, L.: Growth optimal portfolio under proportional transaction costs with obligatory diversification. In: Proc. CDC08 (2008). doi:10.1109/CDC.2008.4739164

4. Dynkin, E.B.: Markov Processes, vols. I, II. Academic Press, San Diego (1965) 
5. Irle, A., Sass, J.: Good portfolio strategies under transaction costs: a renewal theoretic approach. In: Stochastic Finance, pp. 321-341. Springer, New York (2006)

6. Karatzas, I., Schreve, S.: Brownian Motion and Stochastic Calculus. Springer, Berlin (1991)

7. Khasminski, R.Z.: Stochastic Stability of Differential Equations. Sijthoff \& Noordhoff, Rockville (1980)

8. Kushner, H.J., Dupuis, P.: Numerical Methods for Stochastic Control Problems in Continuous Time. Springer, New York (2001)

9. Mackiavicius, V.: Passage to the limit in problems of optimal stopping of Markov processes. Litov. Mat. Sb. 13, 115-128 (1973)

10. Morton, A.J., Pliska, S.R.: Optimal portfolio management with fixed transaction costs. Math. Finance 5, 337-356 (1995)

11. Platen, E., Heath, D.: A Benchmark Approach to Quantitative Finance. Springer, Berlin (2006)

12. Robin, M.: Controle impulsionnel des processus de Markov. Thesis, University of Paris IX (1978)

13. Robin, M.: On some impulse control problems with long run average cost. SIAM J. Control Optim. 19, 333-358 (1981)

14. Stettner, $Ł .:$ Discrete time risk sensitive portfolio optimization with consumption and proportional transaction costs. Appl. Math. 32, 395-404 (2005)

15. Stettner, Ł.: Long time growth optimal portfolio with transaction costs. Banach Cent. Publ. 83, 231241 (2008)

16. Stettner, Ł.: Penalty method for finite horizon stopping problems. Submitted (2008)

17. Tamura, T.: Maximizing the growth rate of a portfolio with fixed and proportional transaction costs. Appl. Math. Optim. 54, 95-116 (2006)

18. Tamura, T.: Maximization of the long-term growth rate for a portfolio with fixed and proportional transaction costs. Adv. Appl. Probab. 40, 673-695 (2008)

19. Scheffé, H.: A useful convergence theorem for probability distributions. Ann. Math. Stat. 18, 434-438 (1947)

20. Williams, D.: Probability with Martingales. Cambridge University Press, Cambridge (1991) 